\title{
Suizidwunsch bei Menschen mit einer psychischen Erkrankung: Symptom oder autonomer Entscheid?
}

Paul Hoff

Korrespondenz:

Prof. Dr. med. Dr. phil. Paul Hoff Psychiatrische Universitätsklinik Zürich

Lenggstrasse 31

Postfach 1931

CH-8032 Zürich

paul.hoff[at]puk.zh.ch
Autonomie ist aus gutem Grund ein zentraler Begriff der medizinischen Ethik. Entscheidungen sollen aufgrund zuverlässiger Informationen und nach sorgfältiger Abwägung getroffen und verantwortet werden. Dies gilt nicht nur für die Entscheidungssituation des Patienten und der Patientin, sondern auch für das ärztliche Handeln. Dieser Grundtenor bildet sich in den (keineswegs nur psychiatrisch relevanten) aktuellen Konzepten von Alliance, Empowerment und Recovery ab. All dies ist sehr hilfreich für die diagnostische und therapeutische Arbeit. Und doch: Nicht alle Fragen können so beantwortet, nicht alle Probleme so gelöst werden, vor allem nicht die ethisch heikelsten: In der Psychiatrie sind dies die Anwendung von Zwang und der Umgang mit Suizidalität.

Aktuell überschneiden sich bezüglich der Frage der Suizidbeihilfe bei psychisch Kranken zwei im Grunde völlig getrennte Fragen:

1. Kann es überhaupt ärztliche Aufgabe sein, einem sterbewilligen Menschen unter bestimmten Bedingungen Beihilfe zum Suizid zu leisten? Die Antwort fällt bekanntlich sehr unterschiedlich aus, sowohl in der Ärzteschaft wie in der Bevölkerung. Aber jede mögliche Antwort muss, wenn sie nicht diskriminierend sein will, für alle Personen in gleicher Weise gelten. Das bedeutet: Wer grundsätzlich unter klar bestimmten Voraussetzungen die ärztliche Beihilfe zur Selbsttötung für erlaubt, ja für ge-
Die Schweizerische Akademie der Medizinischen Wissenschaften (SAMW) hat 2004 medizin-ethische Richtlinien zur «Betreuung von Patientinnen und Patienten am Lebensende» veröffentlicht. Anfragen an die Zentrale Ethikkommission (ZEK) der SAMW haben gezeigt, dass in Einzelfällen eine nicht vertretbare Praxis der ärztlichen Suizidhilfe besteht, und zwar teilweise mit, teilweise aber auch ohne Beteiligung einer Sterbehilfeorganisation. Die ZEK hat dies zum Anlass genommen, in einer Stellungnahme auf die in den Richtlinien aufgeführten Kriterien hinzuweisen und die Voraussetzungen für deren Einhaltung zu präzisieren. Die Stellungnahme wurde in der SÄZ vom 14. März 2012 veröffentlicht und durch kurze Standpunkte aus einer individuell-fachlichen Perspektive ergänzt. Der dritte und letzte Standpunkt beleuchtet das Thema aus einem psychiatrischen Blickwinkel.

tischen Menschen, den quälende imperative Stimmen zum sofortigen Suizid drängen, stellt den einen Pol dar. Den Gegenpol repräsentiert eine Person, die zwar mehrfach im Leben eine schwere depressive Episode durchgemacht hat, jedoch nun weitgehend remittiert ist und aufgrund einer akuten Lebenskrise bei Arbeitslosigkeit und Trennungssituation einen dezidierten Sterbewunsch äussert. Genau hier - bei

\section{Geht es um einen Bilanzsuizid als zu respektierende autonome Entscheidung, oder belegt die Suizidalität lediglich den durch die psychische Erkrankung bedingten markanten Verlust an Autonomie?}

boten hält, kann nicht allein qua Diagnose psychisch Kranke ausschliessen. Hierbei geht es nicht um ein psychiatrisches Problem, sondern um ärztliches Selbstverständnis (und Standesrecht) sowie um gesellschaftliche Grundhaltungen zum Suizid selbst und damit auch zur Beihilfe zum Suizid.

2. Anders bei der zweiten Frage: Wie wird ärztlicherseits der Sterbewunsch einer psychisch kranken Person verstanden? Der Spannungsbogen ist beträchtlich: Der Sterbewunsch eines akut psycho- der Frage des Zusammenhanges zwischen Sterbewunsch und psychischer Erkrankung - liegt das eigentliche Problem. Pointiert formuliert: Geht es im Einzelfall um einen Bilanzsuizid als zu respektierende autonome Entscheidung? Oder belegt, gerade umgekehrt, die Suizidalität lediglich den durch die psychische Erkrankung bedingten markanten Verlust an Autonomie?

Wir werden dieses gravierende Problem nicht durch den blossen Verweis auf begriffliche Dichoto- 
mien wie «urteilsfähig vs. urteilsunfähig» oder «psychotisch vs. nicht psychotisch» lösen können. Das Wesen einer psychischen Erkrankung liegt ja gerade darin, dass sie gleichzeitig ganz unterschiedliche psychische Bereiche in Mitleidenschaft ziehen kann: Eine depressive Person ist nicht einfach traurig, sie hat auch kognitive Beeinträchtigungen, etwa eine Denkhemmung, und - im jetzigen Zusammenhang entscheidend - ihre gesamte Lebensperspektive, vor allem ihre Wertmassstäbe, verändern sich. Wer sich aus einer schwer depressiven Verstimmung heraus dazu entscheidet, eine geplante Berufsausbildung nicht zu beginnen, tut dies auf markant anderer Grundlage, als wenn er oder sie in ausgeglichener Stimmung wäre. Analoges gilt für den Wunsch zur Selbsttötung.

Psychische Erkrankungen führen im Verlauf typischerweise $\mathrm{zu}$ ausgeprägten Schwankungen des Befindens. Dies hat gravierende Auswirkungen auf die Entscheidungsprozesse der betreffenden Person. Auch ein Suizidwunsch, selbst wenn er im Erstkontakt wohlüberlegt wirkt und stimmig vorgetragen wird, kann am nächsten Tag bei geänderter affektiver Grundlage abgeschwächt, anders akzentuiert oder gar verschwunden sein.
Wir sprechen hier von den anspruchsvollsten Aufgaben in der Psychiatrie. Ein praxisnahes Beispiel ist der chronisch depressive Patient, der sich seit kurzem mit der Diagnose eines prognostisch ungünstigen Malignoms konfrontiert sieht und nun um Unterstützung bei seinem Sterbewunsch bittet. Hier braucht es

- Zeit (ein einziges Gespräch kann nicht reichen);

- die Bereitschaft, sich unvoreingenommen auf die Lebens- und Wertewelt des Betroffenen einzulassen (eigene weltanschauliche Positionen müssen temporär zurücktreten können);

- psychiatrische Erfahrung (wer noch nie eine schwer depressive Person ausführlich untersucht und behandelt hat, kann für den in unserem Beispiel zu fällenden Entscheid nicht hinreichend kompetent sein).

Psychisch kranke Menschen sind selbstverständlich und ohne jede Einschränkung Personen. Sie haben vom Grundsatz her dieselben Rechte und Pflichten wie alle anderen Personen, was niemand bestreiten wird. Aber sie sind eben auch psychisch kranke Personen. Dies wiederum bedingt eine spezifische, auf diesen Personenkreis zugeschnittene Herangehensweise - nicht etwa um Grundrechte einzuschränken,

\section{Wer bestimmt im Einzelfall die Grenze, von der an Urteilsunfähigkeit vorliegt?}

Zwar kann man die ganze Problematik ein wenig grob auf die (rechtliche) Frage der Urteilsfähigkeit reduzieren, aber das hilft nicht wirklich: Denn wer bestimmt im Einzelfall die Grenze, von der an Urteilsunfähigkeit vorliegt? Sicher nicht das Gesetz, denn seine Aufgabe ist es, den Rahmen vorzugeben. Den Inhalt dieses Rahmens können nur die ärztliche Untersuchung und Einschätzung festlegen. Grundlage ist dabei vor allem der sorgfältige psychopathologische Befund. sondern, im Gegenteil, um die Möglichkeit zu schaffen, Grundrechte in verantwortlicher Weise praktisch anwendbar zu machen.

Ein letzter Punkt: Grundlegende Fragen der Gesellschaft, wie etwa das Menschenbild, das wir unserem staatlichen und individuellen Handeln zugrunde legen, müssen breit diskutiert und entschieden werden. Es gibt keine Spezialisten, an die sie abschliessend delegiert werden könnten. Unsere Einstellung zum Suizid ist keine psychiatrische Frage. 